AC 2011-5: AN INSTRUCTIONAL PROCESSOR DESIGN USING VHDL AND AN FPGA

Ronald J. Hayne, The Citadel

Ronald J. Hayne, PhD, is an Assistant Professor in the Department of Electrical and Computer Engineering at The Citadel. His professional areas of interest are digital systems and hardware description languages. He is a retired Army Colonel with experience in academics and Defense laboratories. 


\title{
An Instructional Processor Design using VHDL and an FPGA
}

\begin{abstract}
Most modern processors are too complex to be used as an introductory design example. Many digital design courses and texts use hardware description language models of processors, but they are often ad hoc. What is needed is a basic processor with sufficient complexity, that can be modified, programmed, and tested.

An instructional processor has been developed for use as a design example in an Advanced Digital Systems course. The architecture is separated into teachable subsets. The data path contains the registers and interconnecting busses, while the controller implements the fetch, decode, and execute sequences. The VHDL model of the system can be simulated to demonstrate operation of the processor.
\end{abstract}

The instructional processor is now in its second iteration with an updated controller design and a new microcontroller extension. Results from student homework assignments indicate that they are able to successfully design modifications to the processor and demonstrate their function via simulation. The project continues to achieve its goal as a valuable instructional tool.

\section{Introduction}

Teaching Advanced Digital Systems involves use of many design examples including counters, registers, arithmetic logic units, and memory. The design of a computer processor combines these components into an integrated digital system. Most modern commercial microprocessors are too complex to be used as an introductory example of processor design. Hardware description language models of these processors exist, but are often ad hoc and don't divide the

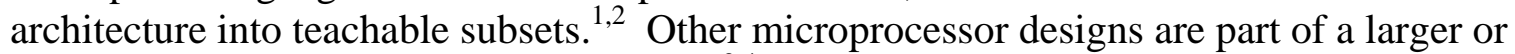
follow-on course in computer architecture. ${ }^{3,4}$ What is needed is a basic processor with sufficient complexity to illustrate major design elements, that can be modified, programmed, and tested.

An instructional processor has been developed for use as an integrated design example in an Advanced Digital Systems course at The Citadel. The architecture is separated into the data path and a sequential controller. The data path contains the memory, registers, arithmetic logic unit (ALU), and interconnecting busses, based on models developed throughout the course. The controller implements the fetch, decode, and execute sequences, using basic state machine design techniques. The entire system is modeled in VHDL and can be simulated to demonstrate operation of the processor. A field programmable gate array (FPGA) implementation also provides a functional hardware version of the processor.

\section{Instruction Set Architecture}

The instruction set architecture of the example processor has been designed to illustrate multiple operations and basic addressing modes. It is based on a three bus organization of a 16-bit data path with a four word register file (REGS). ${ }^{5}$ Key registers include: program counter (PC), 
instruction register (IR), memory data register (MDR), and memory address register (MAR). The basic processor has a 64 word by 16-bit memory (MEM) for storing both programs and data. The complete data path is shown in Figure 1.

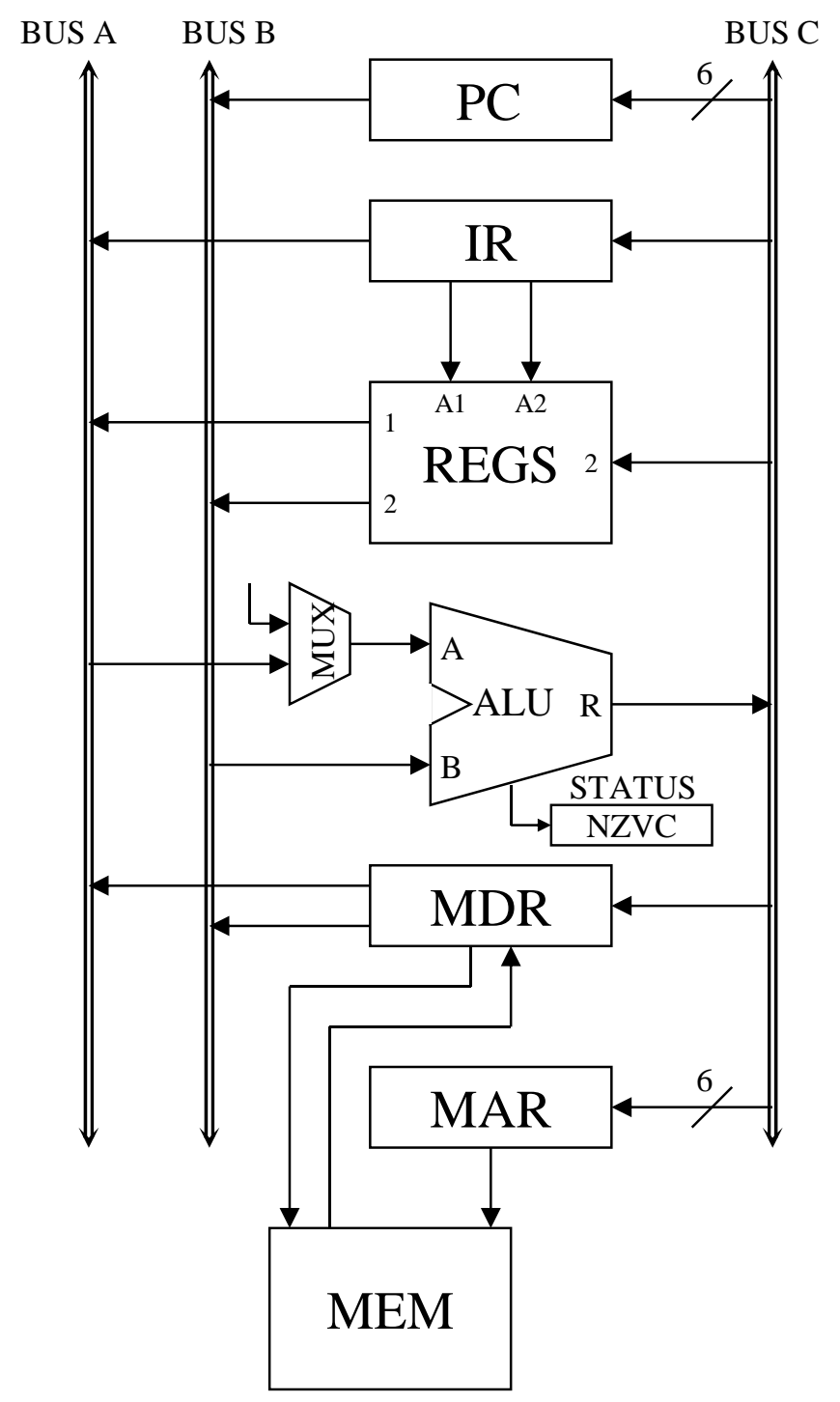

Figure 1. Data Path for Instructional Processor.

The initial implementation of the instructional processor includes opcodes for move (MOVE), add (ADD), and conditional branch (BGTZ), with the capability to modify or supplement these instructions. Access to operands from registers and memory includes provisions for direct (absolute), indirect, and immediate addressing modes. The resulting instruction format contains fields for the opcode (OP), operand source (SRC), and operand destination (DST), as shown in Figure $2 .^{5}$ 


\begin{tabular}{|c|c|c|c|}
\hline & 1211109 & 876 & 5433210 \\
\hline OP & SRC & DST & LUE \\
\hline
\end{tabular}

\begin{tabular}{|l|c|c|l|l|l|}
\hline & Mode & REG \# & \multicolumn{1}{|c|}{ Name } & Syntax & Effective Address \\
\hline \multirow{4}{*}{ SRC } & 00 & $00-11$ & Register Direct & Rn & EA = Rn \\
\cline { 2 - 6 } & 01 & $00-11$ & Register Indirect & $(\mathrm{Rn})$ & EA = [Rn] \\
\cline { 2 - 6 } & 10 & $\mathrm{XX}$ & Immediate & \#Value & Operand = Value \\
\cline { 2 - 6 } & 11 & $\mathrm{XX}$ & Absolute & Value & EA = Value \\
\hline
\end{tabular}

\begin{tabular}{|c|c|c|l|l|l|}
\hline & Mode & REG \# & \multicolumn{1}{|c|}{ Name } & Syntax & Effective Address \\
\hline \multirow{2}{*}{ DST } & 0 & $00-11$ & Register Direct & Rn & EA = Rn \\
\cline { 2 - 6 } & 1 & $\mathrm{XX}$ & Absolute & Value & EA = Value \\
\hline
\end{tabular}

\begin{tabular}{|c|c|l|l|}
\hline OP & Fn & \multicolumn{1}{|c|}{ Assembly Language } & Register Transfer Notation \\
\hline 000 & MOVE & MOVE SRC, DST & DST $\leftarrow$ SRC \\
\hline 001 & ADD & ADD SRC, DST & DST $\leftarrow$ SRC + DST \\
\hline$\ldots$ & & & \\
\hline 110 & BGTZ & BGTZ DISP & PC $\leftarrow$ PC + DISP $(>0)$ \\
\hline 111 & HALT & HALT & Stop $\leftarrow 1$ \\
\hline
\end{tabular}

Figure 2. Processor Instruction Format.

The instruction set and addressing modes have been chosen to provide a basis to illustrate fundamental programming concepts. These include data transfer, counting, indexing, and looping. A simple assembly language program, shown in Figure 3, calculates the sum of an array of numbers to demonstrate these concepts. The example programs are translated into machine code and loaded into memory for testing.

$$
\begin{aligned}
& \text { MOVE N, R1 } \\
& \text { MOVE \#NUM1, R2 } \\
& \text { MOVE } \# \odot, R \odot \\
& \text { LOOP ADD (R2), RO } \\
& \text { ADD \#1, R2 } \\
& \text { ADD \#-1, R1 } \\
& \text { BGTZ LOOP } \\
& \text { MOVE R๑, SUM } \\
& \text { HALT }
\end{aligned}
$$

Figure 3. Assembly Language Program.

Design of the instructional processor is taught in sections covering the instruction set architecture, followed by implementation of the data path, and finally the fetch, decode and execute sequences for the control unit. Each component is modeled in VHDL and functionally verified using ModelSim. ${ }^{6}$ Student homework assignments then involve modification of the 
VHDL model to implement additional instructions. The upgraded processor is verified by the students via execution of their own test programs.

\section{Control Unit Design}

The control unit for the instructional processor is a hardwired controller which generates control signals using a control step counter and the various fields from the instruction register. ${ }^{5}$ The organization of the control unit is shown in Figure 4. The unit is designed as a finite state machine which implements the fetch, decode, and execute sequences for the instruction set architecture.

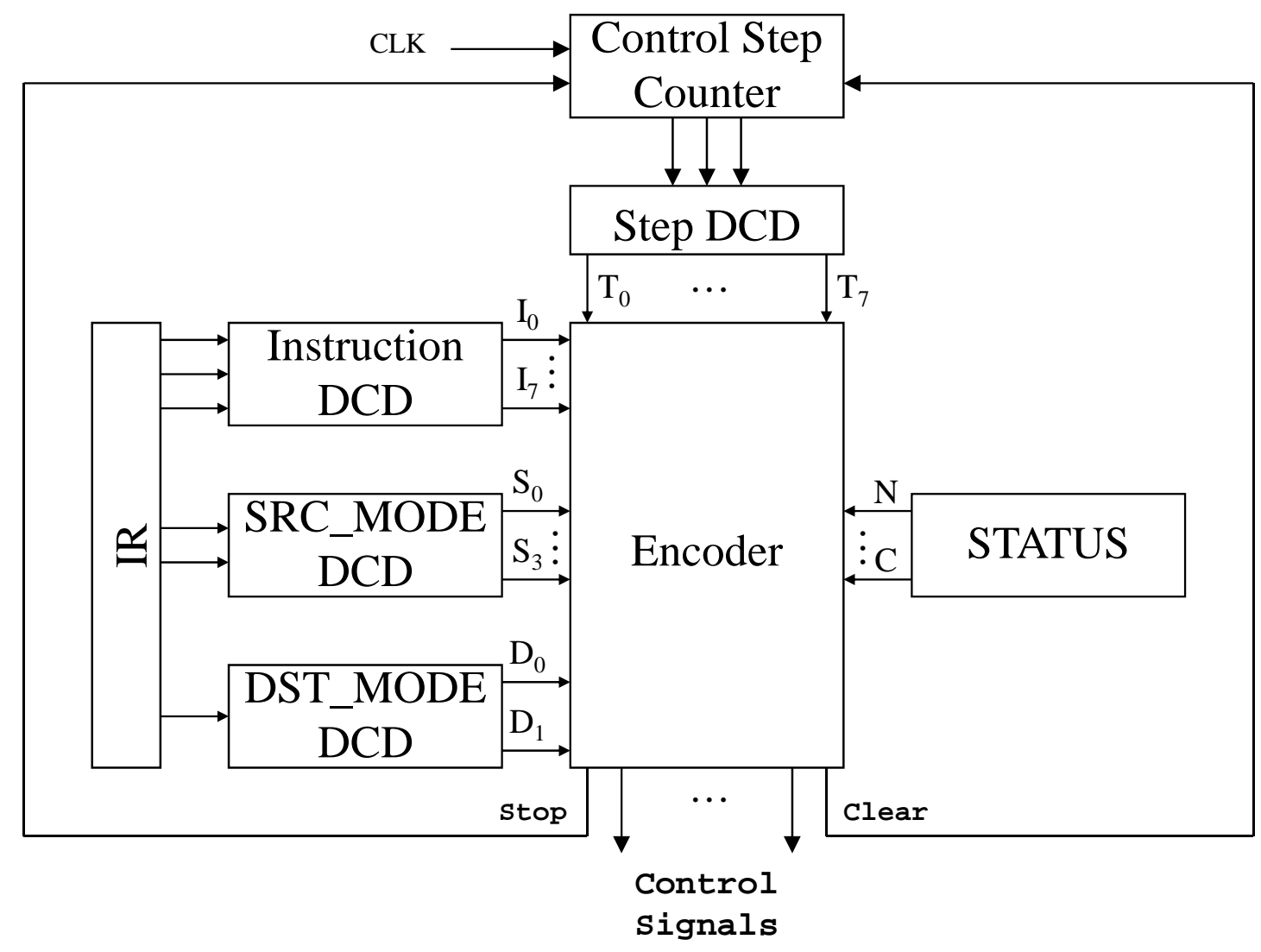

Figure 4. Control Unit Organization.

The first phase of the controller design includes the instruction fetch from memory into the instruction register. The sequence of steps is determined by the control step counter, T0 through T2. The required operations are written in register transfer notation (RTN) and translated into the appropriate control signals, as shown in Figure 5. The instruction is then decoded and an execute sequence is determined for each combination of opcode and addressing mode. A sample instruction, MOVE Rs, Rd, uses register direct addressing for the source (S0), and register direct addressing for the destination (D0). The RTN and control signals for this instruction are shown in Figure 6. 


\begin{tabular}{|l|l|l|}
\hline Step & RTN & Control Signals \\
\hline T0 & MAR $\leftarrow$ PC, PC $\leftarrow$ PC +1 & $\begin{array}{l}\text { BUS_B <= PC } \\
\text { ALU_OP <= Pass_B } \\
\text { Load_MAR <= '1' } \\
\text { Inc_PC <= '1' }\end{array}$ \\
\hline T1 & MDR $\leftarrow$ MEM(MAR) & $\begin{array}{l}\text { MEM_Read <= '1' } \\
\text { Load_MDR <= '1' }\end{array}$ \\
\hline T2 & IR $\leftarrow$ MDR & $\begin{array}{l}\text { BUS_B <= MDR } \\
\text { ALU_OP <= Pass_B } \\
\text { Load_IR <= '1' }\end{array}$ \\
\hline
\end{tabular}

Figure 5. Instruction Fetch Sequence.

\begin{tabular}{|l|l|l|}
\hline Step & RTN & Control Signals \\
\hline T3 & $\mathrm{R}(\mathrm{D}) \leftarrow \mathrm{R}(\mathrm{S})$ & REGS_Read1 $<=$ ' $^{\prime}$ \\
& & ALU_OP <= Pass_A \\
& & Load_STATUS <= '1' \\
& & REGS_Write <= '1' \\
& & Clear <= '1' \\
\hline
\end{tabular}

Figure 6. Instruction Execute for MOVE Rs, Rd.

\section{VHDL Model}

The VHDL model for the instructional processor is developed in phases, with new capabilities added in each phase. Phase 1 includes the components of the data path, which have been developed throughout the course. These include the memory, registers and ALU shown in Figure 1. A portion of the VHDL data path is shown in Figure 7.

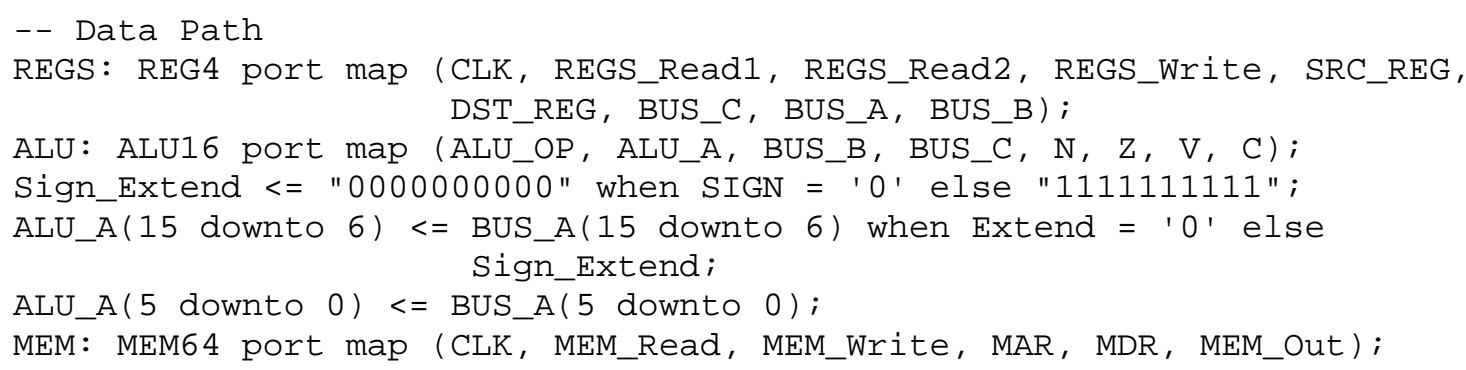

Figure 7. VHDL Model for Data path.

Implementation of the controller follows the state machine design techniques used in many previous examples in the course. The VHDL model follows directly from the control signals derived in the previous section. A portion of the control unit implementing the instruction fetch sequence from Figure 5 is shown in Figure 8. The execute sequence for the MOVE instruction in Figure 6 is also shown in Figure 9. 


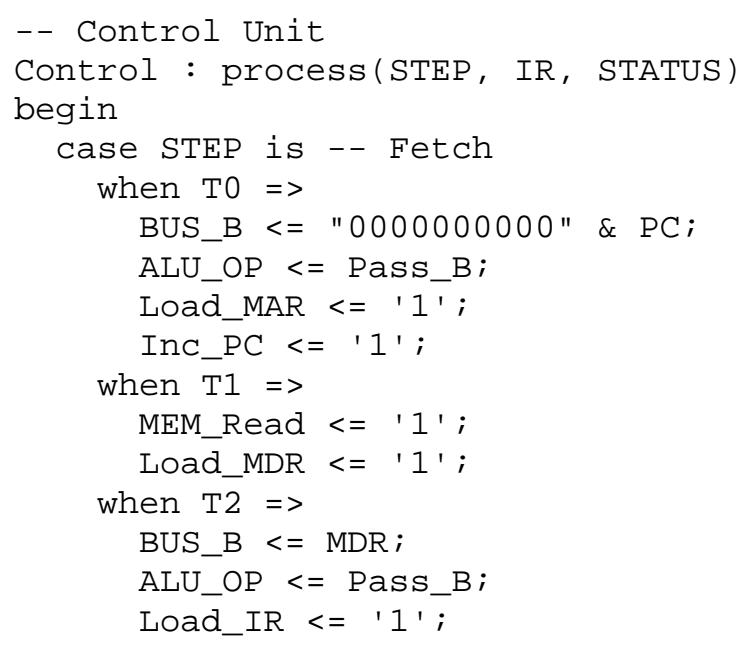

Figure 8. VHDL Model for Control Unit Fetch.

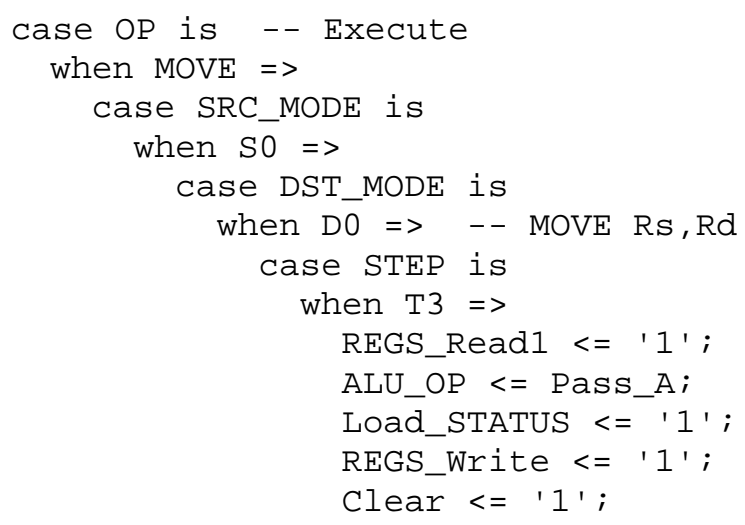

Figure 9. VHDL Model for Control Unit Execute.

Functional verification of the VHDL model is accomplished via simulation using sample assembly language programs translated into machine code. The program is loaded into memory as part of the VHDL model as shown in Figure 10. Simulation with ModelSim produces the waveform shown in Figure 11. This waveform demonstrates the correct fetch and execute sequence for the Phase 1 data transfer instructions.

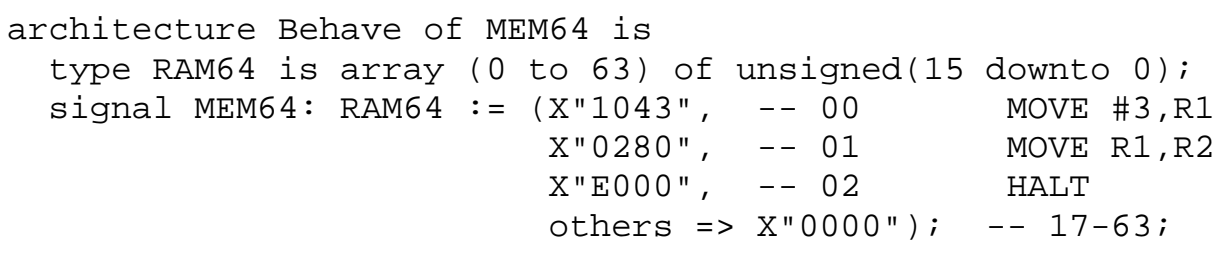

Figure 10. VHDL Model for Memory. 


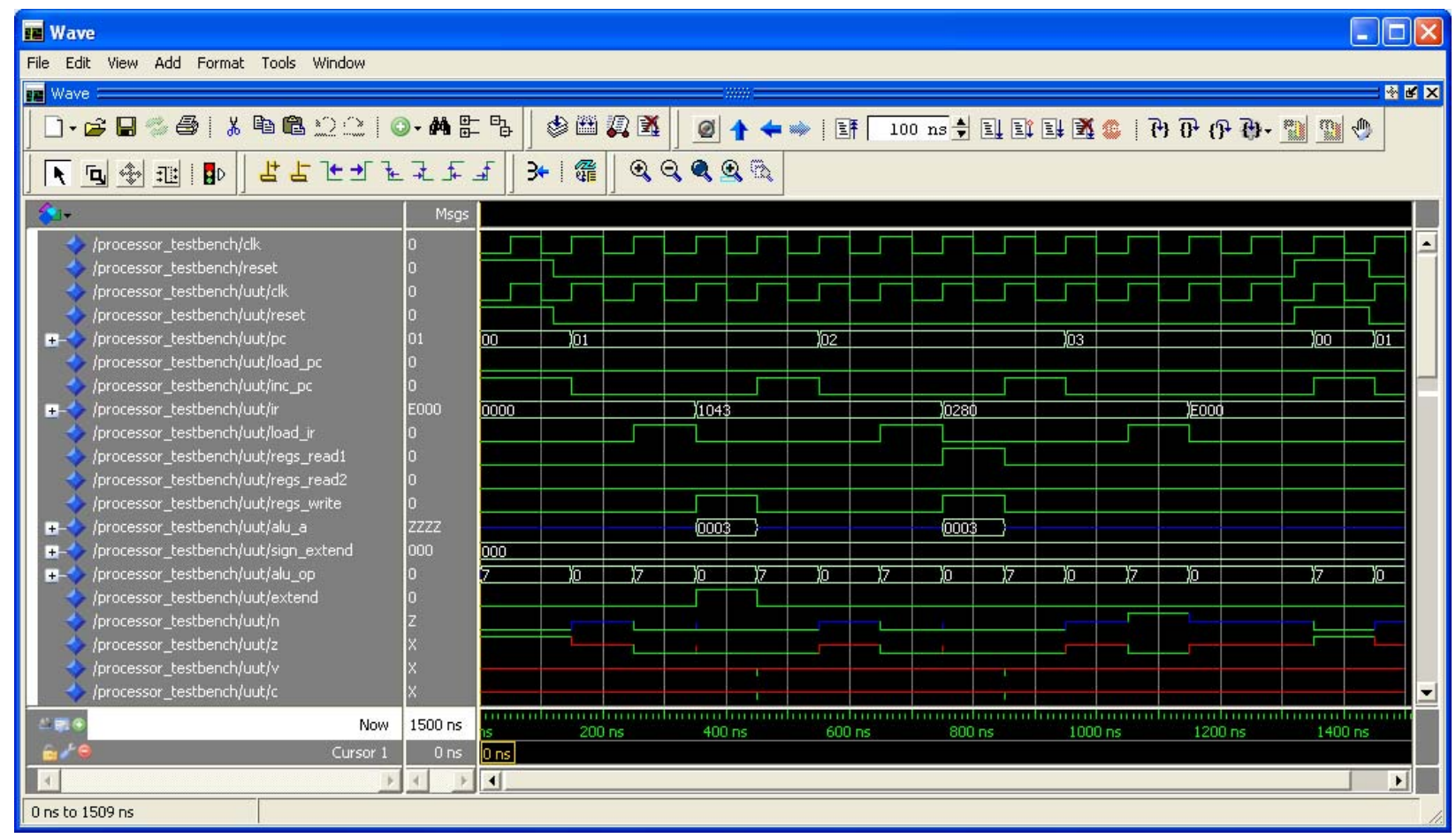

Figure 11. VHDL Simulation Waveform.

Phase 2 of the design process includes development of execution sequences for the remaining instructions and addressing modes necessary to implement the sample assembly language program from Figure 3. This example program is sufficient to demonstrate fundamental programming concepts such as counting, indexing, and looping. The resulting processor is again verified via functional simulation using ModelSim. While the instructional processor is now capable of executing the sample program, a number of instructions and addressing modes are still incomplete and can serve as student homework exercises.

\section{Student Homework Assignments}

Student homework assignments involve expansion of the instructional processor by adding new addressing mode and opcode combinations. For example the assembly language instruction MOVE (Rs ), Rd uses register indirect addressing for the source (S1) and register direct addressing for the destination (D0). Students must first determine the correct RTN sequence necessary to execute the instruction. Then, they translate the RTN sequence into the appropriate control signals for the data path.

The VHDL model for the execute sequence for the added instructions follows directly from the RTN and control signals developed by the students. By adding their VHDL sequence to the instructional processor model provided by the professor, the students then have a functional model of the processor which can be tested via simulation. Students encode their assembly language instructions into machine code and load them into the VHDL memory model. The entire model is then simulated with ModelSim to verify correct functioning of the students new instructions. 
Students can also add new branch instructions to the processor. The HALT instruction from the Phase 1 model can be readily replaced by a branch always (BRA DISP) command. This provides an alternate method for stopping a program by creating an infinite loop. Unlike the data manipulation instructions, these relative branch instructions modify the program counter to alter the execution sequence of the program. Development of the RTN and control signals leads directly to a new execute sequence for the VHDL model.

\section{Microcontroller Extension}

Phase 3 of the processor design involves creation of a basic microcontroller by adding simple memory-mapped input and output to the processor. An 8-bit parallel input port (PORTA) and an 8-bit parallel output port (PORTB) are mapped to fixed locations in the memory address space. The VHDL model for the memory is easily modified to accommodate the new ports. Users can then interface with external devices using the existing data transfer instructions.

The microcontroller extension also includes addition of several new instructions: logic and (AND), logic invert (INV), and rotate left (ROTL). The expanded instruction set is now sufficient to create timing loops with the ability to produce various flashing and shifting patterns on output LEDs. User inputs via switches provide the ability to interact with the processor and select specific output sequences.

The VHDL model of the microcontroller was synthesized using the Xilinx ISE design tools. ${ }^{7}$ The resulting hardware model is mapped to a Xilinx Spartan 3e FPGA on a Digilent Basys board. $^{8}$ PORTA of the microcontroller is mapped to eight input switches on the board, while PORTB is mapped to eight LEDs. The final hardware implementation, shown in Figure 12, utilizes less than 25\% of the FPGA resources, leaving plenty of room for further expansion.

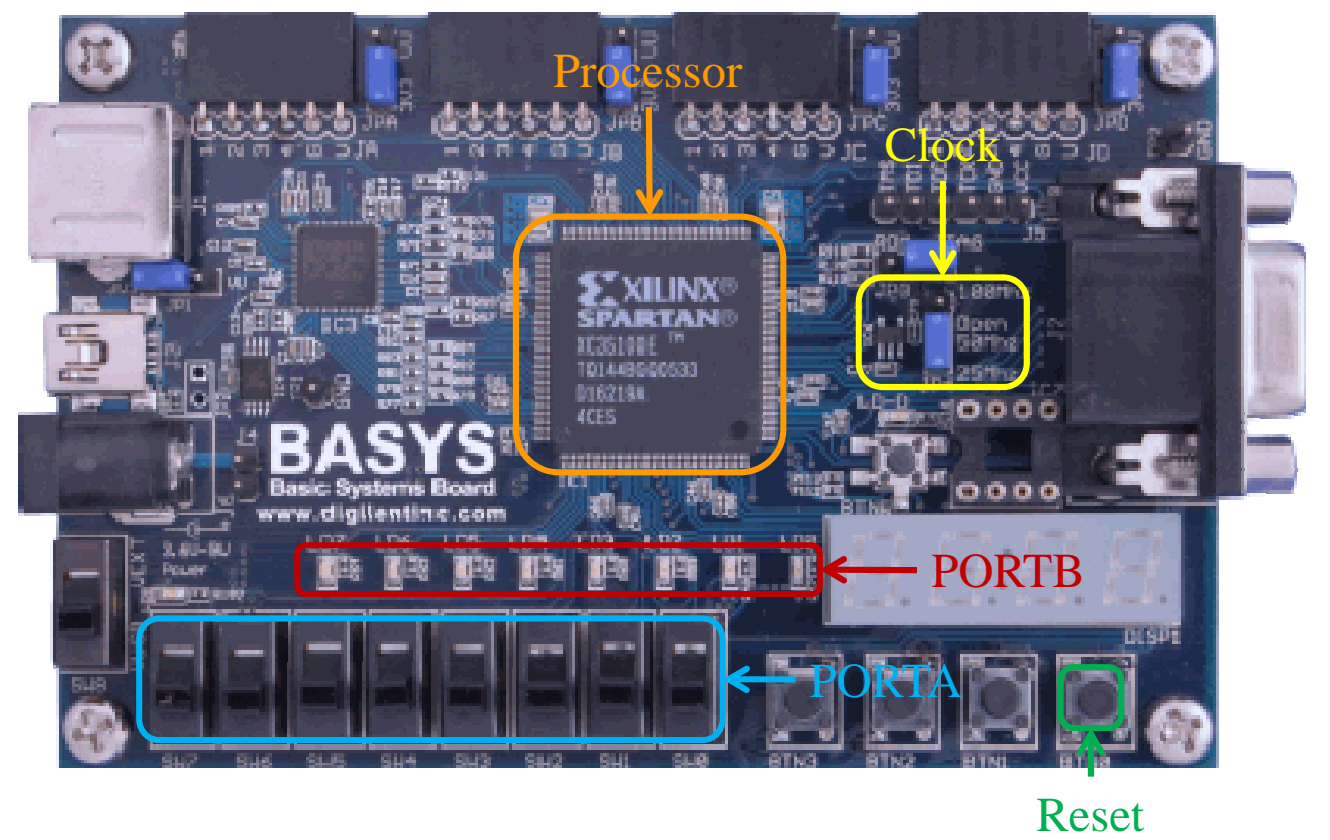

Figure 12. FPGA Implementation of Microcontroller. 


\section{Results and Conclusions}

Implementation of the instructional processor is now in its second iteration with an updated controller design and the new microcontroller extension. The processor is used as a design example to replace the existing MIPS microprocessor in the current course text. ${ }^{1}$ Because the new design uses examples from throughout the semester, it integrates directly into the flow of the course. Development of the model in phases allows separate coverage of the data path and the sequential controller. Design of the control unit is further subdivided into the fetch sequence and execute sequences for instructions as they are added to the processor.

Realization of only a subset of the processor instructions provides sufficient capabilities to demonstrate fundamental programming concepts such as data transfer, counting, indexing, and looping. Additional instructions are implemented as student homework assignments allowing direct application of the design techniques taught in class. Student feedback is very positive that the processor illustrates basic design concepts without unnecessary complexity. Results from homework submissions indicate that the students are able to successfully design modifications to the processor and demonstrate their functionality via simulation.

Addition of the new microcontroller extension provides the final link between the processor model and actual hardware. Simulation exercises are sufficient for functional demonstrations and homework assignments, but the FPGA implementation puts processor hardware in the hands of the students. Inclusion of memory-mapped input and output allows integration with external devices like switches and LEDs providing an interactive presentation of the microcontroller system. The complete project, instruction set architecture, VHDL model, software and hardware, continues to achieve its goal as a valuable instructional tool.

\section{Bibliography}

1. Roth, C. and L. John, Digital Systems Design Using VHDL, Second Edition, Thompson, Toronto, Canada, 2008.

2. Lee, S., Advanced Digital Logic Design: Using VHDL, State Machines, and Synthesis for FPGAs, Thompson, Toronto, Canada, 2007.

3. Harris, D.M. and S.L. Harris, Digital Design and Computer Architecture, Morgan Kaufmann, San Francisco, CA, 2007.

4. Hwang, E.O., Digital Logic and Microprocessor Design with VHDL, Thompson, Toronto, Canada, 2006.

5. Hayne, R.J., "VHDL Projects to Reinforce Computer Architecture Classroom Instruction," Computers in Education Journal, Vol. XVIII No. 2, April - June 2008.

6. ModelSim PE Student Edition, Mentor Graphics Corp., 2010.

7. Xilinx ISE 10.1i Software Manuals, Xilinx, Inc., 2008.

8. Digilent Basys Board Reference Manual, Digilent Inc., 2007. 